\title{
SOME STABILITY PROPERTIES OF ARENS REGULAR BILINEAR OPERATORS
}

\author{
by A. ÜLGER
}

(Received 23rd January 1990)

\begin{abstract}
In this paper we present three results about Arens regular bilinear operators. These are: (a). Let $X, Y$ be two Banach spaces, $K$ a compact Hausdorff space, $\mu$ a Borel measure on $K$ and $m: X \times Y \rightarrow \mathbb{C}$ a bounded bilinear operator. Then the bilinear operator $\tilde{m}: C(K, X) \times C(K, Y) \rightarrow \mathbb{C}$ defined by $\tilde{m}(\phi, \psi)=\int_{K} m(\phi(t),(\psi(t)) d \mu(t)$ is regular iff $m$ is regular. (b) Let $\left(X_{\alpha}\right),\left(X_{\alpha}\right),\left(Z_{z}\right)$ be three families of Banach spaces and let $m_{\alpha}: X_{\alpha} \times Y_{a} \rightarrow Z_{a}$ be a family of bilinear operators with $\sup _{\alpha}\left\|m_{\alpha}\right\|<\infty$. Then the bilinear operator $m:\left(\sum_{\alpha} \oplus X_{a}\right)_{1} \times\left(\sum_{\alpha} \oplus Y_{\alpha}\right)_{1} \rightarrow$ $\left(\sum_{a} \oplus Z_{a}\right)_{1}$ defined by $m\left(\left(x_{a}\right),\left(y_{a}\right)\right)=\left(m_{a}\left(x_{a}, y_{a}\right)\right)$ is regular iff each $m_{a}$ is regular. (c) Let $X, Y$ have the Dieudonné property and let $m: X \times Y \rightarrow Z$ be a bounded bilinear operator with $m(X \times Y)$ separable and such that, for each $z^{\prime}$ in ext $Z_{1}^{\prime}, z^{\prime} \circ m$ is regular. Then $m$ is regular. Several applications of these results are also given.
\end{abstract}

1980 Mathematics subject classification (1985 Revision); Primary 47A99, 46H99.

\section{Introduction}

Let $X, Y, Z$ be three Banach spaces. Denote by $X^{\prime}$ and $X^{\prime \prime}$ the continuous first and second duals of $X$ and consider $X$ as naturally embedded into $X^{\prime \prime}$. In [1] R. Arens has shown that given any bounded bilinear operator $m: X \times Y \rightarrow Z$ there exist two bounded bilinear operators $m^{* * *}: X^{\prime \prime} \times Y^{\prime \prime} \rightarrow Z^{\prime \prime}$ and $m^{t^{* * *}}: X^{\prime \prime} \times Y^{\prime \prime} \rightarrow Z^{\prime \prime}$ that extend the operator $m$. When $m^{* * *}=m^{* * * t}$, the bilinear operator $m$ is said to be "Arens regular". This is equivalent to saying that, for each functional $z^{\prime}$ in $Z^{\prime}$, the bilinear form $z^{\prime} \circ m: X \times Y \rightarrow \mathbb{C}$ is weakly compact [22, Theorem 2.2] i.e., the linear operator $u: X \rightarrow Y^{\prime}$ corresponding to $z^{\prime} \circ m$ is weakly compact. In this paper, our purpose is to study some stability properties of Arens regular bilinear operators. To explain the content of the paper, we need some notation. The terminologies unexplained here are explained or referenced in Section 1 below. Let $K$ be a compact Hausdorff space. By $C(K, X)$ we denote the Banach space of the continuous functions $\phi: K \rightarrow X$ equipped with the supremum norm. By $X_{1}$ we denote the closed unit ball of $X$ and by ext $X_{1}^{\prime}$ the set of extreme points of the closed unit ball of $X^{\prime}$. For a family $\left(X_{a}\right)_{a \in I}$ of Banach spaces, by $\left(\sum_{a} \oplus X_{a}\right)_{p}(1<p<\infty)$ we denote their $l^{p}$-sum. Now let $m: X \times Y \rightarrow \mathbb{C}$ be a bilinear form and $\mu$ a positive Borel measure on $K$. To $m$ and $\mu$ associate the bilinear form $\tilde{m}: C(K, X) \times C(K, Y) \rightarrow \mathbb{C}$ defined by $\tilde{m}(\phi, \psi)=\int_{K} m(\phi(t), \psi(t)) d \mu(t)$. The main result of Section 2 states that the bilinear form $\tilde{m}$ is Arens regular iff $m$ is. Several variants and applications of this result are 
given. Next, let $\left(X_{\alpha}\right),\left(Y_{\alpha}\right),\left(Z_{\alpha}\right)(\alpha \in I)$ be three families of Banach spaces and, for each $\alpha$ in $I$, let $m_{\alpha}: X_{\alpha} \times Y_{\alpha} \rightarrow Z_{\alpha}$ be a bounded bilinear operator such that $\sup _{\alpha}\left\|m_{\alpha}\right\|<\infty$. Define the bilinear operator

$$
m:\left(\sum_{\alpha} \oplus X_{\alpha}\right)_{1} \times\left(\sum_{\alpha} \oplus Y_{\alpha}\right)_{1} \rightarrow\left(\sum_{\alpha} \oplus Z_{\alpha}\right)_{1}
$$

by $m\left(\left(x_{\alpha}\right),\left(y_{\alpha}\right)\right)=\left(m_{\alpha}\left(x_{\alpha}, y_{\alpha}\right)\right)$. The main result of Section 3 states that the bilinear operator $m$ is Arens regular iff each operator $m_{\alpha}$ is. As an application of this result, we give a very short proof of the known fact that the direct sum algebra of a family of Banach algebras $\left(A_{\alpha}\right) \alpha \in I$ is Arens regular iff each factor algebra $A_{\alpha}$ is Arens regular [2, Theorem 6]. In Section 4 our primary concern is the following question: Let $m: X \times Y \rightarrow Z$ be a bounded bilinear operator. Suppose that, for each $z^{\prime}$ in ext $Z_{1}^{\prime}$, the bilinear form $z^{\prime} \circ m$ is Arens regular. Is then $m$ Arens regular? The main result of Section 4 states that if $X$ and $Y$ both have the Dieudonne property [12] and $m(X \times Y)$ is separable then the answer to the preceding question is affirmative. As an application of this result, we give a very short proof of the known fact that, for an algebra $A$ not containing an isomorphic copy of $l^{1}$, the algebra $C(K, A)$ is Arens regular iff $A$ is so [24]. The main ingredients of the proofs are a result of $J$. Diestel [7] about weak compactness in the space $L^{1}(\mu, X)$ of Bochner integrable functions, some results of $A$. Grothendieck [12] and N. J. Kalton, E. Saab and P. Saab [15] about the Dieudonné property, and Choquet's integral representations Theorem [19].

\section{Notation and preliminaries}

In this section, we shall explain some of the notation and terminology mentioned in the introduction and introduce some others. Our notation and terminology are standard and match those of the books $[8,11]$ with the one exception that we denote the continuous dual of a Banach space $X$ by $X^{\prime}$ instead of $X^{*}$. By a linear (or bilinear) operator we shall always mean a continuous linear (or bilinear) operator. For a linear operator $u$, by $u^{*}$ and $u^{* *}$ we denote its first and second adjoints. We recall that a bilinear operator $m: X \times Y \rightarrow Z$ is Arens regular iff for each $h$ in $Z^{\prime}$ and each of the sequences $\left(x_{i}\right)$ in $X_{1}$ and $\left(y_{j}\right)$ in $Y_{1}$

$$
\lim _{i} \lim _{j}\left\langle h, m\left(x_{i}, y_{j}\right)\right\rangle=\lim _{j} \lim _{i}\left\langle h, m\left(x_{i}, y_{j},\right)\right\rangle
$$

whenever these limits exist, see for instance [22, Cor. 2.5]. For a family $\left(X_{\alpha}\right)_{\alpha \in I}$ of Banach spaces, their $l^{p}$-sum $(1<p<\infty)$ and $c_{0}$-sum are defined, respectively, as

$$
\left(\sum_{\alpha} \oplus X_{\alpha}\right)_{p}=\left\{x=\left(x_{\alpha}\right): x_{\alpha} \in X_{\alpha} \text { and }\|x\|_{p}=\left\{\sum_{\alpha}\left\|x_{\alpha}\right\|^{p}\right\}^{1 / p}<\infty\right\}
$$




$$
\left(\sum_{\alpha} \oplus X_{\alpha}\right)_{\infty}=\left\{x=\left(x_{\alpha}\right): x_{\alpha} \in X_{\alpha} \text { and }\|x\|_{\infty}=\sup _{\alpha}\left\|x_{\alpha}\right\|<\infty\right\}
$$

and

$$
\left(\sum_{\alpha} \oplus X_{\alpha}\right)_{0}=\left\{x=\left(x_{\alpha}\right): x_{\alpha} \in X_{\alpha}, \forall \varepsilon>0 \quad\left\|x_{\alpha}\right\|<\varepsilon \text { for all but finitely many } \alpha\right\} .
$$

Equipped with the norm $x \rightarrow\|x\|_{p}(1<p<\infty)$ (resp: $\left.x \rightarrow \sup _{\alpha}\left\|x_{\alpha}\right\|\right)$ the spaces $\left(\sum_{\alpha} \oplus X_{a}\right)_{p}$ and $\left(\sum_{a} \oplus X_{\alpha}\right)_{0}$ are Banach spaces. An element $x^{\prime \prime}$ in the second dual $X^{\prime \prime}$ of a Banach space $X$ is said to be a Baire-1 functional if there exists sequence $\left(x_{n}\right)$ in $X$ such that $x^{\prime \prime}=$ weak $^{*}-\lim x_{n} \cdot B_{1}(X)$, the collection of Baire-1 functionals in $X^{\prime \prime}$, is a closed linear subspace of $X^{\prime \prime}[16]$, see also [17, p. 381]. This notion has been introduced by $A$. Grothendieck in [12, p. 159]. In the same paper A. Grothendieck defines the Dieudonné property and gives several characterizations of this property [12, pp. 158-159]. Among these characterizations we shall take the following as the definition of the Dieudonné property: A Banach space $X$ is said to have the Dieudonné property if, for any Banach space $Y$ and any linear operator $u: X \rightarrow Y$, the inclusion $u^{* *}\left(B_{1}(X)\right) \subseteq Y$ implies that $u$ is weakly compact $[12$, p. 158 , prop. 11 (2 bis)]. As remarked by A. Grothendieck on p. 159 of [12], a Banach space $X$ has the Dieudonné property iff, for any Banach space $Y$, a linear operator $u: X \rightarrow Y$ that sends weakly Cauchy sequences into weakly convergent ones is weakly compact. This characterization is very useful and will be used below. Among the Banach spaces having the Dieudonné property we have the space $C(K)$ for any compact Hausdorff space $K[12$, p. 160 , Theorem 6]; any Banach space $X$ that does not contain an isomorphic copy of $l^{1}$ (this is an immediate consequence of Rosenthal's $l^{1}$-Theorem [20, Main Theorem] and the above characterization of the Dieudonné property); and $C(K, X)$ for any Banach space $X$ that does not contain a copy of $l^{1}[15$, Theorem 4). In [18] A. Pelczynski introduced the so-called $V$-property: A Banach space $X$ has the $V$-property if, for any Banach space $Y$, a linear operator $u: X \rightarrow Y$ that transforms weakly unconditionally Cauchy series into unconditionally convergent ones is weakly compact $[18$, p. 642$]$. Comparing this definition with the above characterization of the Dieudonne property, one can easily see via the Orlicz-Pettis Theorem [8, p. 22], that any Banach space $X$ having the $V$-property also has the Dieudonne property. Several uniform algebras, including the disc algebra [5, p. 293, Cor.], certain Wilken algebras [6, p. 123, Theorem] and the algebras described in Theorem 2.6 of $[25$, p. 144] have the $V$-property and hence also the Dieudonné property.

\section{Lifting Arens regular bilinear operators}

In this section, $X, Y, Z$ are three Banach spaces, $K$ a compact Hausdorff space and $\mu$ a regular Borel measure on $K$. The main result of this section is the following theorem.

Theorem 2.1. Let $m: X \times Y \rightarrow \mathbb{C}$ be a bilinear form. To $m$ and $\mu$ we associate the 
bilinear form $\tilde{m}: C(K, X) \times C(K, Y) \rightarrow \mathbb{C}$ defined by $\tilde{m}(\phi, \psi)=\int_{K} m(\phi(t), \psi(t)) d \mu(t)$. Then $\tilde{m}$ is Arens regular iff $m$ is Arens regular.

Proof. We first remark that the bilinear form $\tilde{m}$ is bounded. Assume $m$ is Arens regular and let $\left(\phi_{i}\right)\left(\right.$ resp. $\left.\left(\psi_{j}\right)\right)$ be a sequence in the unit ball of $C(K, X)(\operatorname{resp} . C(K, Y)$ ) for which the iterated limits

$$
\lim _{i} \lim \tilde{m}\left(\phi_{i}, \psi_{j}\right) \text { and } \lim _{j} \lim _{i} \tilde{m}\left(\phi, \psi_{j}\right)
$$

exist. We have to prove that these limits are equal. Let $u: X \rightarrow Y^{\prime}$ be the linear operator corresponding to $m$, i.e. $m(x, y)=\langle u(x), y\rangle$. The bilinear form $m$ being Arens regular, $u$ is weakly compact [22, Theorem 2.2.]. Let $H$ be the closure of $u\left(X_{1}\right)$. The set $H$ is a weakly compact convex subset of $Y^{\prime}$. Let $L^{1} \mu\left(K, Y^{\prime}\right)$ be the space of Bochner integrable functions $f: K \rightarrow Y^{\prime}$. By a result of J. Diestel [7, Theorem 2], the set $\tilde{H}=\left\{f \in L^{\prime} \mu\left(K, Y^{\prime}\right)\right.$ : for a.e.t. in $K, f(t) \in H\}$ is a relatively weakly compact subset of $L^{1} \mu\left(K, Y^{\prime}\right)$. Now introduce a new bilinear form

$$
T: L^{1} \mu\left(K, Y^{\prime}\right) \times C(K, Y) \rightarrow \mathbb{C}
$$

defined by

$$
T(f, \psi)=\int_{K}\langle f(t), \psi(t)\rangle d \mu(t)
$$

This bilinear form is easily seen to be bounded. Moreover, the sequence $f_{i}=u \circ \psi_{i}$ lies in the set $\tilde{H}$ and

$$
T\left(u \circ \phi_{i}, \psi_{j}\right)=\int_{K}\left\langle u\left(\phi_{i}(t)\right), \psi_{j}(t)\right\rangle d \mu(t)=\int_{K} m\left(\phi_{i}(t), \psi_{j}(t)\right) d \mu(t)
$$

The set $\tilde{H}$ being relatively weakly compact and bilinear form $T$ continuous, Grothendieck's double limit criterion [13, Theorem 6] shows that the above iterated limits are equal. Thus $\tilde{m}$ is Arens regular. To prove the converse, it is enough to apply the double limit criterion to the sequences of constant functions of the forms $\phi_{i}(t) \equiv x_{i}$ and $\psi_{j}(t) \equiv y_{j}$.

We proceed with some corollaries. The first corollary is apparently stronger than the theorem but, in fact, they are equivalent.

Corollary 2.2. Let $m: X \times Y \rightarrow Z$ be a bilinear operator and $g$ a function in $L^{1} \mu\left(K, Z^{\prime}\right)$. To $m, g$ and $\mu$ we associate the bilinear form

$$
T_{g}: C(K, X) \times C(K, Y) \rightarrow \mathbb{C} \text { defined by } T_{g}(\phi, \psi)=\int_{K}\langle g(t), m(\phi(t), \psi(t)\rangle d \mu(t) .
$$

Then, $T_{g}$ is Arens regular if $m$ is. 
Proof. Assume $m$ is Arens regular. As is well-known $L^{1} \mu\left(K, Z^{\prime}\right)=L^{1} \mu(K) \hat{\otimes} Z^{\prime}$, the projective tensor product of $L^{1} \mu(K)$ and $Z^{\prime}\left[8\right.$, p. 228]. The space $L^{1} \mu(K) \hat{\otimes} Z^{\prime}$ being the completion of the algebraic tensor product $L^{1} \mu(K) \otimes Z^{\prime}$, there exists a sequence $\left(g_{n}\right)$ in this latter space that converges in the norm of $L^{1} \mu\left(K, Z^{\prime}\right)$ to $g$. Since, for $\phi$ in $C(K, X)$ and $\psi$ in $C(K, Y)$

$$
\left\|T_{g}(\phi, \psi)-T_{g_{n}}(\phi, \psi)\right\| \leqq\|m\|\left\|g-g_{n}\right\|_{1}\|\phi\|\|\psi\| \rightarrow 0
$$

and since, in the operator norm, the limit of a sequence of weakly compact operators is weakly compact, it is enough to show that $T_{g}$ is Arens regular for each $g$ in $L^{1} \mu(K) \otimes Z^{\prime}$. Now, each element of $L^{1} \mu(K) \otimes Z^{\prime}$ is a finite sum of the form $\sum_{i=1}^{k} \beta_{i} \otimes z_{i}^{\prime}$, where $\beta_{i}$ 's are in $L^{1} \mu(K)$ and $z_{i}^{\prime}$ 's are in $Z^{\prime}$. Therefore it is enough to show that $T_{g}$ is Arens regular for a $g$ of the form $g=\beta \otimes z^{\prime}$. But for such a $g$,

$$
\begin{aligned}
T_{g}(\phi, \psi) & =\int_{K}\left\langle\beta(t) z^{\prime}, m(\phi(t), \psi(t))\right\rangle d(t) \\
& =\int_{K} z^{\prime} \circ m(\phi(t), \psi(t)) \beta(t) d \mu(t) .
\end{aligned}
$$

Now in the theorem, we replace $m$ by $z^{\prime} \circ m$ and the measure $\mu$ by $\beta \mu$ and conclude that $T_{g}$ is Arens regular.

As an application of this result we have the following result.

Corollary 2.3. Let $m: X \times Y \rightarrow Z$ be a bilinear operator and suppose $Z^{\prime}$ has the Radon-Nikodym property. To $m$ associate the bilinear operator $\tilde{m}: C(K, X) \times C(K, Y) \rightarrow C(K, Z)$ defined by $\tilde{m}(\phi, \psi)(t)=m(\phi(t), \psi(t))$. Then $\tilde{m}$ is Arens regular iff $m$ is Arens regular.

Proof. Assume $m$ is Arens regular. We have to show that, for each $\lambda$ in $C(K, Z)^{\prime}$, the bilinear form $\lambda \circ \tilde{m}$ is Arens regular. The elements of $C(K, Z)^{\prime}$ are regular countably additive vector measures of bounded variation from the Borel $\sigma$-algebra of $K$ into $Z^{\prime}[9$, p. 380]. Now let $\lambda$ be an element of $C(K, Z)^{\prime}$ and $\mu=|\lambda|$ be the variation of $\lambda$. Then $\mu$ is a regular countably additive positive Borel measure [8, p.3, Proposition 9]. Since $\lambda$ is absolutely continuous with respect to $\mu$ and $Z^{\prime}$ has the RNP, there exists a function $g$ in $L^{1} \mu\left(K, Z^{\prime}\right)$ representing $\lambda[8$, p. 61 , Definition 3] so that

$$
\lambda \circ \tilde{m}(\phi, \psi)=\int_{\mathbf{K}}\langle g(t), m(\phi(t), \psi(t))\rangle d \mu(t)
$$

It follows from the preceding corollary that $\tilde{m}$ is Arens regular. The converse is immediate.

As an application of this corollary we have the following result.

Corollary 2.4. Let $X$ be a reflexive Banach space, $K(X)$ the space of compact linear 
operators on $X$ and $m: X \times X \rightarrow X$ a bilinear operator. To $m$ associate the bilinear operator $\tilde{m}: K(X) \times K(X) \rightarrow K(X)$ defined by $\tilde{m}(u, v)(x)=m(u(x), v(x))$. Then $m$ is Arens regular.

Proof. Let $S$ be the closed unit ball of $X$ equipped with the weak topology. We embed $K(X)$ into $C(S, X)$ in a natural way and introduce the bilinear operator $\hat{m}: C(S, X) \times C(S, X) \rightarrow C(S, X)$ defined by $\hat{m}(\phi, \psi)(s)=m(\phi(s), \psi(s))$. Since any reflexive space has the RNP, by the preceding corollary, $\hat{m}$ is Arens regular. Since $\tilde{m}$ is the restriction of $\hat{m}$ to $K(X) \times K(X), \tilde{m}$ is also Arens regular.

\section{Direct sum of bilinear operators}

In this section $\left(X_{\alpha}\right),\left(Y_{\alpha}\right),\left(Z_{\alpha}\right)(\alpha \in I)$ are three families of Banach spaces and $m_{\alpha}: X_{\alpha} \times$ $Y_{\alpha} \rightarrow Z_{\alpha}(\alpha \in I)$ is a family of bilinear operators. We assume that $\sup _{\alpha}\left\|m_{\alpha}\right\|<\infty$. To the family $\left(m_{\alpha}\right)$ we associate the bilinear operator.

$$
m:\left(\sum_{\alpha} \oplus X_{\alpha}\right)_{1} \times\left(\sum_{\alpha} \oplus Y_{\alpha}\right)_{1} \rightarrow\left(\sum_{\alpha} \oplus Z_{\alpha}\right)_{1}
$$

defined by $m\left(\left(x_{\alpha}\right),\left(y_{\alpha}\right)\right)=\left(m_{\alpha}\left(x_{\alpha}, y_{\alpha}\right)\right)$. This is a well-defined bounded bilinear operator. The main result of this section is the following theorem.

Theorem 3.1. The bilinear operator $m$ is Arens regular iff each bilinear operator $m_{\alpha}$ is Arens regular.

Proof. Assume each $m_{\alpha}$ is Arens regular. Let $h=\left(h_{\alpha}\right)$ be an element of $\left(\sum_{\alpha} \oplus Z_{\alpha}^{\prime}\right)_{\infty}$, the dual space of $\left(\sum_{\alpha} \oplus Z_{\alpha}\right)_{1}$. Then $h \circ m=\left(h_{\alpha} \circ m_{\alpha}\right)$. Let $u:\left(\sum_{\alpha} \oplus X_{\alpha}\right)_{1} \rightarrow\left(\sum_{\alpha} \oplus Y_{\alpha}^{\prime}\right)_{\infty}$ and $u_{\alpha}: X_{\alpha} \rightarrow Y_{\alpha}^{\prime}$ be, respectively, the linear operators corresponding to $h \circ m$ and $h_{\alpha} \circ m_{\alpha}$. The operator $u$ and the $u_{\alpha}$ 's satisfy $u\left(\left(x_{\alpha}\right)\right)=\left(u_{\alpha}\left(x_{\alpha}\right)\right)$ for $\left(x_{\alpha}\right)$ in $\left(\sum_{\alpha} \oplus X_{\alpha}\right)_{1}$. As

$$
\sup _{\alpha}\left\|h_{\alpha} \circ m_{\alpha}\right\| \leqq \sup _{\alpha}\left\|h_{\alpha}\right\| \sup _{\alpha}\left\|m_{\alpha}\right\| \leqq\|h\|_{\infty} \cdot \sup _{\alpha}\left\|m_{\alpha}\right\|<\infty
$$

and each element $x=\left(x_{\alpha}\right)$ is absolutely summable, the element $u(x)=\left(u_{\alpha}\left(x_{\alpha}\right)\right)$ actually lies in $\left(\sum_{\alpha} \oplus Y_{\alpha}^{\prime}\right)_{0}$. For the same reason, $u^{*}$ maps $\left(\sum_{\alpha} \oplus Y_{\alpha}^{\prime \prime}\right)_{1}$ into $\left(\sum_{\alpha} \oplus X_{\alpha}^{\prime}\right)_{0}$. It follows that $u^{* *}$ maps $\left(\sum_{\alpha} \oplus X_{\alpha}^{\prime \prime}\right)_{1}$ into $\left(\sum_{\alpha} \oplus Y_{\alpha}^{\prime \prime}\right)_{1}^{\prime}$. Moreover, as one can see very easily, for $x^{\prime \prime}=\left(x_{\alpha}^{\prime \prime}\right)$ in $\left(\sum_{\alpha} \oplus X_{\alpha}^{\prime \prime}\right)_{1}, u^{* *}\left(x^{\prime \prime}\right)=\left(u_{\alpha}^{* *}\left(x_{\alpha}^{\prime \prime}\right)\right)$. Now let $x^{\prime \prime}=x_{1}^{\prime \prime}+\xi$ be an element of $\left(\sum_{\alpha} \oplus X_{\alpha}\right)^{\prime \prime}$ decomposed according to the following well-known decomposition

$$
\left(\sum_{\alpha} \oplus X_{\alpha}\right)_{1}^{\prime \prime}=\left(\sum_{\alpha} \oplus X_{\alpha}^{\prime \prime}\right)_{1} \oplus\left(\sum_{\alpha} \oplus X_{\alpha}^{\prime}\right)_{0}^{\perp}
$$

Then, for each $y^{\prime \prime}$ in $\left(\sum_{\alpha} \oplus Y_{\alpha}^{\prime \prime}\right)_{1}$, 


$$
\left\langle u^{* *}\left(x^{\prime \prime}\right), y^{\prime \prime}\right\rangle=\left\langle u^{* *}\left(x_{1}^{\prime \prime}\right), y^{\prime \prime}\right\rangle+\left\langle u^{* *}(\xi), y^{\prime \prime}\right\rangle=\left\langle u^{* *}\left(x_{1}^{\prime \prime}\right), y^{\prime \prime}\right\rangle
$$

since $u^{*}\left(y^{\prime \prime}\right)$ is in $\left(\sum_{\alpha} \oplus X_{\alpha}^{\prime}\right)_{0}$. Thus we have the equality

$$
u^{* *}\left(x^{\prime \prime}\right)=u^{* *}\left(x_{1}^{\prime \prime}\right)
$$

Next, by Arens regularity of $m_{\alpha}, u_{\alpha}$ being weakly compact [22, Theorem 2.2], $u_{a}^{* *}$ applies $X_{\alpha}^{\prime \prime}$ into $Y_{\alpha}^{\prime}$. It follows that, for $x_{1}^{\prime \prime}=\left(x_{\alpha}^{\prime \prime}\right)$ in $\left(\sum \oplus X_{\alpha}^{\prime \prime}\right)_{1}, u^{* *}\left(x_{1}^{\prime \prime}\right)=\left(u_{\alpha}^{* *}\left(x_{\alpha}^{\prime \prime}\right)\right)$ lies in $\left(\sum_{\alpha} \oplus Y_{\alpha}^{\prime}\right)_{0}$. Thus, by $\left(^{*}\right), u^{* *}$ maps the second dual of $\left(\sum_{\alpha} \oplus X_{\alpha}\right)_{1}$ into $\left(\sum_{\alpha} \oplus Y_{\alpha}^{\prime}\right)_{0}$. This proves that $u$ is weakly compact, and $m$ is Arens regular. For the converse it is enough to remark that the restriction of $m$ to $X_{\alpha} \times Y_{\alpha}$ is $m_{\alpha}$.

The proof of the preceding theorem shows that, when each $m_{a}$ is Arens regular, for $x^{\prime \prime}=x_{1}^{\prime \prime}+\xi$ and $y^{\prime \prime}=y_{1}^{\prime \prime}+\eta$ in

$$
\left(\sum_{\alpha} \oplus X_{\alpha}\right)_{1}^{\prime \prime}=\left(\sum_{\alpha} \oplus X_{\alpha}^{\prime \prime}\right)_{1} \oplus\left(\sum_{\alpha} \oplus X_{\alpha}^{\prime}\right)_{0}^{\perp}
$$

and

$$
\left(\sum_{\alpha} \oplus Y_{\alpha}\right)_{1}^{\prime \prime}=\left(\sum_{\alpha} \oplus Y_{\alpha}^{\prime \prime}\right)_{1} \oplus\left(\sum_{\alpha} \oplus Y_{\alpha}^{\prime}\right)_{0}^{\perp}
$$

respectively, we have

$$
m^{* * *}\left(x^{\prime \prime}, y^{\prime \prime}\right)=m^{* * *}\left(x_{1}^{\prime \prime}, y_{1}^{\prime \prime}\right)
$$

As an application of this theorem we give the next corollary. We recall that a Banach algebra $A$ is said to be "Arens regular" if on the second dual $A^{\text {" of }} A$ the two Arens products coincide $[3,10]$. This is equivalent to saying that, for each $a^{\prime}$ in $A^{\prime}$, the bilinear form $m$ on $A \times A$ defined by $m(a, b)=\left\langle a^{\prime}, a b\right\rangle$ is Arens regular. Now let $\left(A_{\alpha}\right)_{\alpha \in I}$ be a family of Banach algebras and $A=\left(\sum_{\alpha} \oplus A_{\alpha}\right)_{1}$. Then $A$, equipped with the coordinatewise multiplication, is a Banach algebra. As an immediate corollary of the preceding theorem and the remark following it we have the following result, which is Theorem 6 of [2]. At this point we point out that the proof of the preceding theorem in essence is a generalization of the proof of [2, Theorem 6].

Corollary 3.2. The algebra $A=\left(\sum_{\alpha} \oplus A_{\alpha}\right)_{1}$ is Arens regular iff each algebra $A_{\alpha}$ is Arens regular. Moreover, in this case, the unique Arens product of $A^{\prime \prime}$ is given, for $a^{\prime \prime}=\left(a_{a}^{\prime \prime}\right)+\xi$ and $b^{\prime \prime}=\left(b^{\prime \prime}=\left(b_{a}^{\prime \prime}\right)+\eta\right.$ in

$$
A^{\prime \prime}=\left(\sum_{\alpha} \oplus A_{\alpha}^{\prime \prime}\right)_{1} \oplus\left(\sum_{\alpha} \oplus A_{\alpha}^{\prime}\right)_{0}^{\perp}
$$

by $a^{\prime \prime} \cdot b^{\prime \prime}=\left(a_{a}^{\prime \prime} \cdot b_{a}^{\prime \prime}\right)$. 


\section{Extreme point regularity}

In this section, $X, Y, Z$ will be three Banach spaces and $K$ a compact Hausdorff space. By ext $Z_{1}^{\prime}$, we shall denote the set of extreme points of $Z_{1}^{\prime}$, and $B_{1}(X)$ the subspace of $X^{\prime \prime}$ consisting of Baire-1 functionals. We assume that all our vector spaces are defined over the field of real numbers $\mathbb{R}$. This assumption is not essential, but it will simplify some notation.

Let $m: X \times Y \rightarrow Z$ be a linear operator. Let us say that $m$ is "extreme point regular" if, for $z^{\prime}$ in ext $Z_{1}^{\prime}$, the bilinear form $z^{\prime} \circ m$ is Arens regular. The main question of this section is this: Does extreme point regularity imply Arens regularity? Although a positive answer to this question seems to be unlikely, we do not have any counterexample to it. Below we show that at least in two important cases the answer to this question is positive.

Proposition 4.1. If the space $Z$ does not contain a copy of $l^{1}$ then every extreme point regular bilinear operator $m: \mathrm{X} \times Y \rightarrow \mathrm{Z}$ is Arens regular.

Proof. This is an immediate consequence of a result of $R$. Haydon [14, p. 269], which says that if $Z$ does not contain a copy of $l^{1}$ then, in the norm topology of $Z^{\prime}$, the convex hull of ext $Z_{1}^{\prime}$ is dense in $Z_{1}^{\prime}$.

As an application of this proposition, we give the following result due to $N$. Young [26, Theorem 3], see also [23, Corollary to Theorem 2].

Corollary 4.2. Let $A=K(X)$ be the operator algebra of compact linear operators on $X$. Then $A$ is Arens regular iff $X$ is reflexive.

Proof. Assume $X$ is reflexive. Then the algebra $A$ does not contain a copy of $l^{1}[4$, Corollary 1.12], and ext $A_{1}^{\prime}=\operatorname{ext} X_{1}^{\prime} \otimes \operatorname{ext} X_{1}$ [21, Theorem 1.3]. Now, for $u, v$ in $A$ and $x^{\prime} \otimes x$ in ext $A_{1}^{\prime}$,

$$
\left\langle u \circ v, x^{\prime} \otimes x\right\rangle=\left\langle u^{*}\left(x^{\prime}\right), v(x)\right\rangle
$$

The space $X$ being reflexive, a simple application of the double limit criterion shows that the bilinear operator $m(u, v)=u \circ v$ from $A \times A$ into $A$ is extreme point regular, so regular. Hence $A$ is Arens regular. The converse is also easy and proved as in $N$. Young's paper [26].

Another important case where extreme point regularity implies Arens reglarity is given in the theorem below. For the proof of this theorem some preparation is needed. In $[12$, p. 161] A. Grothendieck remarks that a separable Banach space $X$ has the Dieudonné property iff every sequence $\left(x_{n}^{\prime}\right)$ that converges to zero for $\sigma\left(X^{\prime}, B_{1}(X)\right)$ converges weakly to zero. No published proof of this fact being available, the referee has asked us to include a proof of this fact.

Lemma 4.3. A separable Banach space $X$ has the Dieudonne property iff every $\sigma\left(X^{\prime}, B_{1}(X)\right)$ null sequence $\left(x_{n}^{\prime}\right)$ in $X^{\prime}$ is weakly null. 
Proof. (i) Assume $X$ has the Dieudonné property and let $\left(x_{n}^{\prime}\right)$ be a $\sigma\left(X^{\prime}, B_{1}(X)\right)$ null sequence in $X^{\prime}$. Following the referee's suggestion, we introduce the linear operator $T: X \rightarrow c_{0}$ defined by $T(x)=\left(\left\langle x_{n}^{\prime}, x\right\rangle\right)$. By the closed graph theorem, $T$ is continuous. As $\left(x_{n}^{\prime}\right)$ is $\sigma\left(X^{\prime}, B_{1}(X)\right)$-null, $T^{* *}$ maps $B_{1}(X)$ into $c_{0}$. Hence, since $X$ has the Dieudonne property, $T^{* *}$ maps $X^{\prime \prime}$ into $c_{0}$, and $\left(x_{n}^{\prime}\right)$ is weakly null.

(ii) Conversely, assume the condition of the lemma is satisfied. Let $Y$ be any Banach space and $u: X \rightarrow Y$ a linear operator such that $u^{* *}\left(B_{1}(X)\right)$ is contained in $Y$. We have to show that $u$ is weakly compact. Since the space $u(X)$ is separable, replacing $Y$ by $\overline{u(X)}$ we can and do assume that $Y$ is separable. Then $\left(Y_{1}^{\prime}\right.$, weak $\left.{ }^{*}\right)$ is a metrizable compact space. Let $\left(y_{n}^{\prime}\right)$ be a sequence in $Y_{1}^{\prime}$. Passing to a subsequence if necessary, we can assume that $\left(y_{n}^{\prime}\right)$ converges weak* to an element $y^{\prime}$ in $Y_{1}^{\prime}$. The condition $u^{* *}\left(B_{1}(X)\right) \subseteq Y$ implies that $u^{*}$ is continuous from $\left(Y^{\prime}\right.$, weak $\left.{ }^{*}\right)$ into $\left(X^{\prime}, \sigma\left(X^{\prime}, B_{1}(X)\right)\right.$. Hence $u^{*}\left(y_{n}^{\prime}\right) \rightarrow u^{*}\left(y^{\prime}\right)$ for $\sigma\left(X^{\prime}, B_{1}(X)\right)$. Now the condition of the lemma implies that $u^{*}\left(y_{n}^{\prime}\right) \rightarrow$ $u^{*}\left(y^{\prime}\right)$ weakly. Thus $u^{*}$, so $u$, is weakly compact, and $X$ has the Dieudonné property.

We remark that in part (i) of the above proof we did not use the separability of $X$. We shall use this remark in the proof of the next theorem. In the initial version of this theorem, in the hypotheses, we had " $X$ and $Y$ separable" instead of " $m(X \times Y)$ separable". This stronger version has been suggested by the referee.

Theorem 4. Assume both $X$ and $Y$ have the Dieudonne property and let $m: X \times Y \rightarrow Z$ be an extreme point regular bilinear operator with $m(X \times Y)$ separable. Then $m$ is Arens regular.

Proof. Let $Z_{0}$ be a separable subspace of $Z$ that contains the image of $m$. As is well-known [19, pp. 129-130], given any extreme point in the unit ball of $Z_{0}^{\prime}$, there exists a functional $z^{\prime}$ in ext $Z_{1}^{\prime}$ that extends $z_{0}^{\prime}$. Therefore we can and do assume that $Z$ itself is separable. Then the unit ball of $Z^{\prime}$ equipped with the weak ${ }^{*}$ topology is metrizable. The rest of the proof can conveniently be divided into two parts. In the first part, using only the extreme point regularity of $m$, we shall show that, for any $x^{\prime \prime}$ in $B_{1}(X)$ and $y^{\prime \prime}$ in $B_{1}(Y)$, the equality.

$$
m^{* * *}\left(x^{\prime \prime}, y^{\prime \prime}\right)=m^{r^{* * *} t}\left(x^{\prime \prime}, y^{\prime \prime}\right)
$$

holds. In the second part, using the Dieudonné property, we shall show that the equality (1) holds for all $x^{\prime \prime}$ in $X^{\prime \prime}$ and $y^{\prime \prime}$ in $Y^{\prime \prime}$. That is, $m$ is Arens regular.

Let $x^{\prime \prime}$ in $B_{1}(X)$ and $y^{\prime \prime}$ in $B_{1}(Y)$ be given. Then for some sequences $\left(x_{i}\right)$ in $X$ and $\left(y_{j}\right)$ in $Y, x^{\prime \prime}=$ weak $^{*}-\lim x_{i}$ and $y^{\prime \prime}=$ weak $^{*}-\lim y_{j}$. It follows that [1, Theorem 3.2], for any $h$ in $Z^{\prime}$,

$$
\lim _{i} \lim _{j}\left\langle h, m\left(x_{i}, y_{j}\right)\right\rangle=\left\langle h, m^{* * *}\left(x^{\prime \prime}, y^{\prime \prime}\right)\right\rangle
$$




$$
\lim _{j} \lim _{i}\left\langle h, m\left(x_{i}, y_{j}\right)\right\rangle=\left\langle h, m^{i * * * t}\left(x^{\prime \prime}, y^{\prime \prime}\right)\right\rangle
$$

The fact that $m$ is extreme point regular implies that, for $z^{\prime}$ in ext $Z_{1}^{\prime}$, the equality

$$
\left\langle z^{\prime}, m^{* * *}\left(x^{\prime \prime}, y^{\prime \prime}\right)\right\rangle=\left\langle z^{\prime}, m^{t * * * t}\left(x^{\prime \prime}, y^{\prime \prime}\right)\right\rangle
$$

holds. Now fix a functional $h$ in $Z_{1}^{\prime}$. By Choquet's integral representation Theorem [19, pp. $120-127]$, there exists a Borel probability measure $\mu$ on $\left(Z_{1}^{\prime}\right.$, weak $\left.{ }^{*}\right)$ concentrated on ext $Z_{1}^{\prime}$ such that, for all $i$ and $j$,

$$
\left\langle h, m\left(x_{i}, y_{j}\right)\right\rangle=\int_{\text {exi } Z_{1}^{\prime}}\left\langle z^{\prime}, m\left(x_{i}, y_{j}\right)\right\rangle \mathrm{d} \mu\left(z^{\prime}\right)
$$

Taking into account the equalities (2), (3), (4) and applying the Lebesgue Dominated Convergence Theorem to (5) we get the equality

$$
\left\langle h, m^{* * *}\left(x^{\prime \prime}, y^{\prime \prime}\right)\right\rangle=\left\langle h, m^{t * * * t}\left(x^{\prime \prime}, y^{\prime \prime}\right)\right\rangle \text {. }
$$

The functional $h$ being arbitrary, this proves equality (1).

Fix again a functional $h$ in $Z_{1}^{\prime}$. Let $u: X \rightarrow Y^{\prime}$ (resp. $v: Y \rightarrow X^{\prime}$ ) be the linear operators representing the bilinear form $h \circ m$. We have to prove that $u$ is weakly compact. To prove this, since $X$ has the Dieudonne property, it is enough to show that $u^{* *}$ maps $B_{1}(X)$ into $Y^{\prime}$. To this end, let $x^{\prime \prime}$ be an arbitrary element of $B_{1}(X)$. Then $x^{\prime \prime}=$ weak*- $\lim x_{i}$ for some sequence $\left(x_{i}\right)$ in $X$. Let $y^{\prime \prime}$ be an arbitrary element in $B_{1}(Y)$. Then $y^{\prime \prime}=$ weak $^{*}-\lim y_{j}$ for some sequence $\left(y_{j}\right)$ in $Y$. Now, by (6) and [22, Proposition. 2.1]

$$
\begin{aligned}
\lim _{i}\left\langle u\left(x_{i}\right), y^{\prime \prime}\right\rangle & =\lim _{i} \lim _{j}\left\langle u\left(x_{i}\right), y_{j}\right\rangle=\lim _{i} \lim _{j}\left\langle h, m\left(x_{i}, y_{j}\right\rangle\right. \\
& =\left\langle h, m^{* * *}\left(x^{\prime \prime}, y^{\prime \prime}\right)\right\rangle=\left\langle h, m^{i * * * t}\left(x^{\prime \prime}, y^{\prime \prime}\right)\right\rangle=\left\langle x^{\prime \prime}, v^{* *}\left(y^{\prime \prime}\right)\right\rangle \\
& =\left\langle v^{*}\left(x^{\prime \prime}\right), y^{\prime \prime}\right\rangle .
\end{aligned}
$$

Thus, $u\left(x_{i}\right) \rightarrow v^{*}\left(x^{\prime \prime}\right)$ for $\sigma\left(Y^{\prime}, B_{1}(Y)\right)$. Since the space $Y$ has the Dieudonné property, by part (i) of Lemma 4.3 above and the remark preceding the theorem, $u\left(x_{i}\right) \rightarrow v^{*}\left(x^{\prime \prime}\right)$ weakly in $Y^{\prime}$. On the other hand, $u^{* *}$ being weak ${ }^{*}$-continuous, $u\left(x_{i}\right) \rightarrow u^{* *}\left(x^{\prime \prime}\right)$ for $\sigma\left(Y^{\prime \prime \prime}, Y^{\prime \prime}\right)$. It follows that $u^{* *}\left(x^{\prime \prime}\right)=v^{*}\left(x^{\prime \prime}\right)$, and $u^{* *}\left(x^{\prime \prime}\right) \in Y^{\prime}$. Thus $u^{* *}$ maps $B_{1}(X)$ into $Y^{\prime}$. Since $X$ also has the Dieudonne property, from this we conclude that $u$ is weakly compact. Hence $m$ is Arens regular [22, Theorem 2.2].

Next we present an application of this theorem. Let $A$ be a Banach algebra. Then the space $C(K, A)$, equipped with the pointwise multiplication and the supremum norm, is a Banach algebra. Recently Arens regularity of the algebra $C(K, A)$ was completely settled 
in [24]. The result is that the algebra $C(K, A)$ is Arens regular iff $A$ is Arens regular. The proof of this result is quite complicated; here, as an application of the preceding theorem, we give a very short proof of this result in an important particular case.

Corollary 4.4. Assume $A$ does not contain a copy of $l^{1}$. Then the algebra $C(K, A)$ is Arens regular iff $A$ is Arens regular.

Proof. Assume $A$ is Arens regular. Since every separable subalgebra of $C(K, A)$ is isomorphic to a subalgebra of $C(S, B)$, where $S$ is a metrizable quotient of $K$ and $B$ is a separable subalgebra of $A$ [24, Section 2a], and since an algebra is Arens regular iff its separable subalgebras are Arens regular, we can and do assume that $K$ is metrizable and $A$ is separable so that $C(K, A)$ is separable. By [15, Theorem 4], the space $C(K, A)$ has the Dieudonne property. Therefore it is enough to prove that the multiplication $m(\phi, \psi)=\phi \cdot \psi$ of $C(K, A)$ is extreme point regular. Now ext $C(K, A)_{1}^{\prime}=\left\{\delta_{t} \otimes a^{\prime}: t \in K\right.$, $\left.a^{\prime} \in \operatorname{ext} A_{1}^{\prime}\right\}$ [21, Theorem 2.4], where $\delta_{t}$ is the Dirac measure at $t$. For $\phi, \psi$ in $C(K, A)$,

$$
\left\langle m(\phi, \psi), \delta_{t} \otimes a^{\prime}\right\rangle=\left\langle\phi(t) \psi(t), a^{\prime}\right\rangle .
$$

Therefore, by Arens regularity of $A$, we conclude, by the double limit criterion, that $m$ is extreme point regular, so regular. Hence $C(K, A)$ is Arens regular. The converse is trivial.

We end this note with two questions. Let $A$ be a Banach algebra.

(a) Is $B_{1}(A)$ a subalgebra of $A^{\prime \prime}$ under either Arens product of $A^{\prime \prime}$ ?

(b) Characterize those Banach algebras for which the two Arens products of $A^{\prime \prime}$ coincide on $B_{1}(A)$. The proof of Theorem 4.4 shows that, for a separable Banach algebra with the Dieudonne property, this is equivalent to Arens regularity.

Acknlowledgements. This paper has been written while the author was visiting the Department of Mathematical Sciences of The University of Arkansas. He gratefully acknowledges the hospitality of this institution and, in particular, that of its chairman, Professor John Duncan. The author is grateful and expresses his sincere thanks to the referee for this most careful reading of this paper and his numerous remarks and suggestions which have permitted us to improve both the presentation and the content of the paper.

\section{REFERENCES}

1. R. Arens, The adjoint of a bilinear operation, Proc. Amer. Math. Soc. 2 (1951), 839-848.

2. N. Arikan, Arens regularity and reflexity, Quart. J. Math. Oxford Ser. (2) 32 (1981), 383-388.

3. P. Civin and B. Yood, The second conjugate space of a Banach algebra as an algebra, Pacific J. Math. 11 (1961), 847-870.

4. H. S. Collins and W. Ruess, Weak compactness in spaces of compact operators and vector-valued functions, Pacific J. Math. 106 (1983), 45-71. 
5. F. Delbaen, Weakly compact operators on disc algebra, J. Algebra 45 (1977), 284-294.

6. F. Delbaen, The Pelczynski property for some uniform algebras, Studia Math. 64 (1979), 117-125.

7. J. Diestel, Remarks on weak compactness in $L_{1}(\mu, X)$, Glasgow Math. J. 18 (1977), 87-91.

8. J. Diestel and J. J. UhL, JR., Vector measures, Math. Surveys 15 (1977).

9. N. Dinculeanu, Vector Measures (Pergamon Press, Berlin, 1967).

10. J. Duncan and S. A. R. Hosseiniun, The second dual of a Banach algebra, Proc. Roy. Soc. Edinburgh 84A (1979), 309-325.

11. N. Dunford and J. T. Schwartz, Linear Operators, Part I (Interscience, New York, 1958).

12. A. Grothendieck, Sur les applications linéaires faiblement compactes d'espaces du type C(K), Canad. J. Math. 5 (1953), 129-173.

13. A. Grothendieck, Criteres de compacité dans les espaces fonctionels généraux, Amer. $J$. Math. 74 (1952), 168-186.

14. R. Haydon, Some more characterizations of Banach spaces containing $l^{1}$, Math. Proc. Cambridge Philos. Soc. 80 (1976), 269-276.

15. N. J. Kalton, E. SaAb and P. SAab, On the Dieudonné property for $C(K, E)$, Proc. Amer. Math. Soc. 96 (1986), 50-52.

16. R. D. McWilliams, A note on weak sequential convergence, Pacific J. Math. 12 (1962), 333-335.

17. E. Odell and P. Rosenthal, A double-dual characterization of separable Banach spaces containing $l^{1}$, Israel J. Math. 20 (1975), 375-384.

18. A. Pelczynski, Banach spaces on which every unconditionally converging operator is weakly compact, Bull. Acad. Polon. Sci. 10 (1962), 641-649.

19. R. P. Phelps, Integral representations for elements of convex sets, in Studies in Functional Analysis (MAA Studies in Mathematics 21, edited by R. G. Bartle.

20. H. P. Rosenthal, A characterization of Banach spaces containing $l^{1}$, Proc Nat. Acad. Sci. U.S.A. 71 (1974), 2411-2413.

21. W. M. Ruess and Ch.P. Stegall, Extreme points in duals of operator spaces, Math. Ann. 261 (1982), 535-546.

22. A. Ülger, Weakly compact bilinear forms and Arens regularity, Proc. Amer. Math. Soc. 101 (1987), 697-704.

23. A. Ülger, Arens regularity of the algebra $K(X)$, Monatsh. Math. 105 (1988), 313-318.

24. A. Ülger, Arens regularity of the algebra $C(K, A)$, J. London Math. Soc. 42 (1990), 354-364.

25 P. Wostaszcyk, On weakly compact operators from some uniform algebras, Studia Math. 64 (1979), 105-116.

26. N. Young, Periodicity of functionals and representations of normed algebras on reflexive Banach spaces, Proc. Edinburgh Math. Soc. 20 (1976), 99-120.

Department of Mathematics

BoĞAZIÇı UnIVERSITY

80815 BebeK, Istangul

TURKEY 